\title{
The association of fFN testing on hospital admissions for preterm labor ${ }^{*}$
}

\author{
Shilpa Iyer", Thomas McElrath, Petr Jarolim, James Greenberg
}

Brigham and Women's Hospital, Harvard Medical School, Boston, USA

Email: " siyer@partners.org

Received 5 November 2012; revised 7 December 2012; accepted 16 December 2012

\begin{abstract}
Objective: To determine if the use of fetal fibronectin (fFN) testing has affected hospital admissions for preterm labor. Methods: ICD-9 and CPT codes from all admissions to Brigham \& Women's Hospital between January 1, 1995 and December 31, 2010 were evaluated. Data recorded included total deliveries, admissions for preterm labor (PTL) without delivery, length of stay (days) for PTL admissions, preterm deliveries, and number of fFN tests performed. The data was evaluated using a Wilcoxon test of trend and least squares regression. Results: Fetal fibronectin testing was introduced mid-year 2001. As a percentage of total deliveries, the number of admissions for PTL without delivery decreased from $3.97 \%$ in 1995 to $2.16 \%$ in $2010(p<0.01)$ and the number of preterm births decreased from $7.54 \%$ to $6.59 \%(p<0.034)$. The number of hospital days for admissions for PTL without delivery per 10,000 births decreased from 1853 days/10,000 births to 903 days/10,000 $(p<0.001)$ births while the number of fFN tests per 10,000 births increased from 0/10,000 births to 1390/10,000 births $(p<0.0001)$. Increased use of FFN was negatively associated with admissions for PTL without delivery ( $p$ $<0.001$ ). The cost for admissions for PTL without delivery based on hospital charge decreased from $\$ 1,748,796$ to $\$ 785,010$ while the cost of $\mathbf{f F N}$ testing increased from $\$ 0$ to $\$ 152,785$ per year. Conclusion: The introduction of fetal fibronectin testing was associated with a dramatic decrease in hospital admissions for PTL. In addition, there was saving of almost $\$ 1,000,000$ per year in charges associated with these admissions.
\end{abstract}

Keywords: Preterm Labor; Fetal Fibronectin; Cost

\footnotetext{
"The introduction of fetal fibronectin testing was associated with a decrease in hospital admissions for preterm labor.

We have no conflicts of interest to disclose in relation to this paper.

"Corresponding author.
}

\section{INTRODUCTION}

Preterm births continue to be the leading cause of infant mortality in the United States. While the infant mortality rate has plateaued from 2000 until the present, the percentage of preterm births has increased. In 2005, 68.6\% of infant deaths were in preterm infants, an increase from $65.6 \%$ in 2000 [1]. Along with increased rates in prematurity, the cost of health care continued to skyrocket from 2000 to 2005, and continues to increase today. In 2008, health care spending accounted for $16.2 \%$ of the United States Gross Domestic Product, surpassing 2.3 trillion dollars. Hospital care accounted for $31 \%$ of health care spending in 2008 [2]. In the current cost-conscious environment, we are increasingly looking towards costsaving prevention measures. Cost-saving measures include reducing unnecessary hospital admissions.

Fetal fibronectin (fFN) is a high molecular weight glycoprotein produced by fetal cells. It is found at the interface between the chorion and the decidua. In adults, about $1 \%$ of plasma protein is fibronectin with the difference between adult and fetal molecules is the glycosylation of one amino acid in fFN. In 1991 Lockwood et al. demonstrated fFN to be an effective predictor of preterm labor (PTL) in pregnant women with intact membranes, and of preterm birth [3]. In 1997 Peaceman et al. determined a negative predictive value of $99.5 \%$ for delivery within 1 week and $99.2 \%$ for delivery within two weeks [4]. After being approved by the FDA in January 1998 [5], fetal fibronectin testing was instituted into practice in 2001 at Brigham and Women's Hospital. The fFN test was used primarily to rule out false PTL, and help reassure providers by providing an extra piece of data. The primary goal of fFN was to help providers to separate true PTL from false PTL or PTL from other causes of pelvic pain. When fFN testing was instituted, it was postulated that cost savings from decreased admission from PTL would offset the costs of fFN testing and decrease antepartum admissions overall [6]. fFN was to be used to better identify true PTL and guide admissions and reduce inpatient costs by decreasing antepar- 
tum hospital admissions. Our objective in this study was to determine if the use of fetal fibronectin (fFN) testing has reduced hospital admissions for PTL and/or preterm births.

\section{METHODS}

In this study reviewed at all births at Brigham and Women's Hospital from January 1, 1995 through December 31, 2010, \% of preterm births, number of fFN tests, hospital days for antepartum admissions, and the associated costs during the same time period. The hospital electronic database was searched using ICD9 and CPT codes for both cesarean section and vaginal deliveries combined, admissions for PTL with an associated delivery (DRG codes 765, 766, 767, 768, 774 and 775), fFN tests used on a triage or inpatient basis (CPT 82731), and admissions for PTL with no associated delivery (644.03). Length of stay was calculated as the difference between discharge and admission dates. fFN use was not correlated with an indication or reason for presentation to $\mathrm{OB}$ triage. Preterm admission and delivery was defined at gestational age less than 37 weeks. Gestational age was rounded from the best clinical estimate of delivery date. Estimated delivery date was calculated by last menstrual period and/or ultrasound in any trimester based on the clinician's best judgment. The study was categorized as exempt by the Partners' Healthcare Institutional Review Board (IRB).

The above data extracted by ICD9 code search was then analyzed in a SAS 4.0 database (SAS Institute Inc., Cary, NC) using the Wilcox test of trend and least squares regression methods. After noting a significant decrease in preterm deliveries during this time period, we further analyzed number of fFN tests by year with admissions for PTL using logistic regression analysis adjusting for preterm deliveries.

The cost of one antepartum room per night was the room charge for one antepartum bed for Brigham and Women's Hospital in 2010, \$1146. It is difficult to estimate the true cost for a specific room in the hospital setting secondary to payer differences, difference in staffing between services, and transfers to and from the labor floor for monitoring of antepartum patients. The room cost used is our best estimate. The annual cost of fFN testing was estimated at \$132,802 at Brigham and Women's Hospital for 2010. The annualized cost of fFN testing includes all reagents and consumables, proficiency testing, quality control measures, and cost of labor. The annualized cost of fFN testing for 2010 was divided by the number of fFN tests in 2010 and then retrospectively applied to fFN testing for the years 2001-2009. While this process likely underestimates the cost of fFN early on in its implementation, it is our best estimate of the actual cost of one fFN test for our institution and better represents changes over the last 5 years. There was no outpatient costs considered in our study.

\section{RESULTS}

From 1995-2010, the 16 years that bracketed the introduction of fFN, there were 137,358 deliveries, 9309 preterm deliveries, 8446 fFN tests performed, 4331 admissions for PTL without delivery, and 18,537 total hospital days for admissions with a diagnosis of PTL. Figure 1 illustrates trends from 1995-2010 of total births measured in thousands, percentage preterm births, number of fFN tests measured in hundreds, and number of hospital days for PTL without a delivery or ante- partum days measured in hundreds. Total deliveries are presented in thousands in Figure 2 in order to show all the data in one format.

The number of total deliveries per year remained relatively constant from 1995 to 2010 ranging from 7589 to 9707 deliveries. The number of preterm deliveries during this time period was significantly decreased from $7.54 \%$ to $6.59 \%$ ( $p<0.034$ ). As a percentage of total deliveries, the number of admissions for PTL without delivery, or antepartum admissions, was significantly decreased from $3.97 \%$ in 1995 to $2.16 \%$ in 2010 (p < $0.01)$. Figure 1 illustrates that this decrease was associated with the introduction of fFN in 2001. The five year

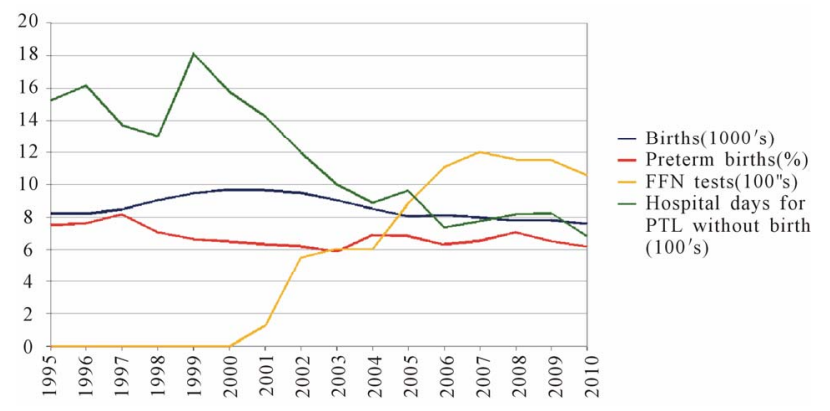

Figure 1. Percentage of preterm births, number of fFN tests, and number of hospital days by year.

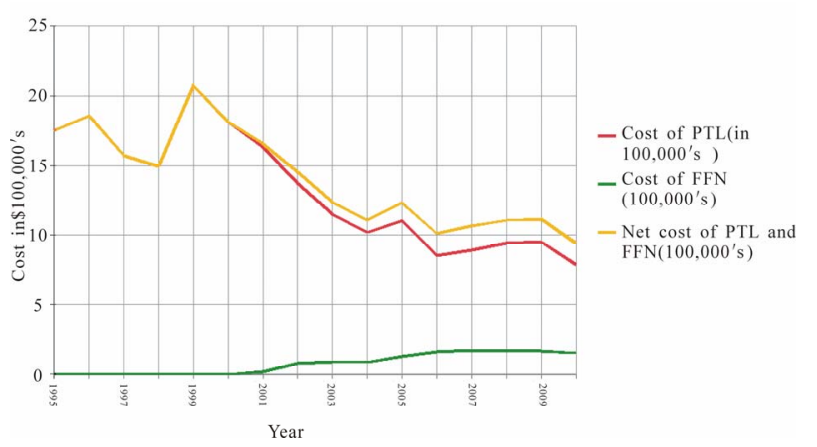

Figure 2. Cost of preterm labor admissions prior to 2001 and cost of preterm labor admissions and fFN tests after 2001. 
average from 1995 to 2000 was $3.98 \%$ whereas the five year average from 2006 to 2010 was $2.57 \%$. The number of hospital days for admissions for PTL without delivery, or antepartum admissions, per 10,000 total births decreased from 1853 days/10,000 births to 903 days/10,000 ( $\mathrm{p}<0.001$ ) births while the number of fFN tests per 10,000 births increased from $0 / 10,000$ births to 1390 / 10,000 births $(\mathrm{p}<0.0001)$ as expected with introduction of fFN testing. Increased use of fFN was negatively associated with admissions for PTL without delivery ( $\mathrm{p}<$ 0.001). Further logistic regression aanalysis was used to adjust for the decrease in preterm delivery and noted significant correlation between fFN use and decreased antepartum admission for PTL $(\mathrm{p}<0.05)$. Figure 1 illustrates that the decreased in inpatient hospital stay for PTL correlates with increasing use of fFN testing.

Based on a daily room hospital charge of \$1146 per day, the cost of admissions for PTL without delivery decreased from \$1,748,796 in 1995 to \$785,010 in 2010. Hospital charges were used in this analysis as they do not vary by insurance status. These costs represent a cost estimate for annual antepartum care for Brigham and Women's Hospital. The hospital charge of fFN testing increased from \$0 in 1995-2000 to \$152,785 in 2010. Additionally, we estimated the total cost of PTL to include the hospital charge for an antepartum beds plus fFN testing from 1995 to 2010. The net cost of PTL decreased from $\$ 1,748,796$ in 1995 to $\$ 937,795$ in 2010. Figure 2 illustrates an overall decrease in cost of preterm labor despite the added cost of fFN testing.

\section{DISCUSSION}

During the 16 years that bracketed fFN introduction, we noted a significant reduction in antepartum admissions for PTL, a significant decrease in the number of antepartum hospital days for PTL, a gradual increasing fFN use, and a significant correlation between increased fFN use and decreasing admissions for PTL. We additionally noted an overall decrease in preterm deliveries. The association between increased fFN use and decreased antepartum admissions and hospital days for PTL remained significant after adjusting for the overall decrease in preterm deliveries using logistic regression analysis.

Our study has the following limitations: 1) The data is cross sectional so that only associations can be made without discussing causation; 2) ICD9 codes were used to gather the data from a large database and expose our study to misclassification bias; 3) We did not review indications for fFN use or more specific reasons for PTL admissions including gestational age at admission. Another study looking at in depth indications for fFN use could provide better guidelines for fFN use and correlation to pregnancy outcomes. Possible confounders to this association include a change in patient demographics, increased use of cervical length measurement, changes in hospital charges, or disincentives for prolonged hospital stays. During the study period time Brigham and Women's Hospital has continued to serve the same catchment area, there were no large changes in hospital charges, and no monetary incentives to decrease prolonged hospital admissions. One limitation of this study is that we did not compare the reasons and specifics for presentation to our triage unit or the changes in numbers of cervical length measurements on the whole as well as per patient. In addition, our data is limited to Brigham and Women's Hospital, a tertiary care center with a large volume of antepartum patients, and may not be relevant to smaller community based hospitals.

Our cost analysis was also limited to Brigham and Women's Hospital with its specialized antepartum care and hospital floors and a large volume of fFN testing. Costs are hospital specific and may be different at a smaller hospital, a different payer mix, or in a different geographic location. Benefits of the study include a large volume of deliveries overall, a large volume of antepartum patients with specialized antepartum care, and a large volume of fFN testing.

We conclude that implementation of fFN testing at Brigham and Women's Hospital is associated with reduced antepartum admissions and reduced costs for PTL. Additionally we showed total reduced costs for PTL since fFN testing became available. This significant decrease in preterm deliveries at Brigham and Women's Hospital reflects national data recently reported by the CDC. In our study we showed a reduced rate of preterm birth compared to national data. Brigham and Women's Hospital is a tertiary referral center, and it is unclear why our preterm birth rates are below the national average. Comparison of patient demographics to national data would be an interesting follow up analysis. While the CDC reported an increase in preterm births from 2000 to 2005, they recently reported a decrease in preterm birth rate from $12.8 \%$ in 2006 to $12.3 \%$ in 2008 [7]. Cost savings while providing effective care is increasingly becoming important during this era of patient affordability and escalating health care costs.

\section{REFERENCES}

[1] MacDorman, M., et al. (2008) Recent trends in infant mortality in the United States. National Center for Health Statistics Data Brief, No. 9.

[2] Kaiser Family Foundation (2009) Health care costs: A primer, key information on health care cost and their impact. http://www.kff.org/insurance/7670.cfm

[3] Lockwood, C., et al. (1991) "Fetal fibronectin in cervical and vaginal secretions as a predictor of preterm delivery. The New England Journal of Medicine, 325, 669-674. 
doi:10.1056/NEJM199109053251001

[4] Peaceman, A.M., et al. (1997) Fetal fibronectin as a predictor of preterm birth in patients with symptoms: A multicenter trial. American Journal of Obstetrics \& Gynecology, 177, 13-18. doi:10.1016/S0002-9378(97)70431-9

[5] US Food and Drug Administration (1998) Report from August 1998 premarket approval.

http://www.fda.gov/MedicalDevices/ProductsandMedical Procedures/DeviceApprovalsandClearances/PMAAppro- vals/ucm115123.htm

[6] Foxman, E.F. and Jarolim, P. (2004) Use of the fetal fibronectin test in decisions to admit to hospital for preterm labor. Clinical Chemistry, 50, 663-665. doi:10.1373/clinchem.2003.028720

[7] Center for Disease Control and Prevention National Center for Health Statistics Data Brief (2010) Are preterm births on the decline in the United States? National Center for Health Statistics Data Brief, No. 39. 